\title{
An Efficient Consistency Algorithm for Solving Tighter Solution Space of Temporal Constraint Optimization Problem
}

\author{
Zhang Wen, Zhu Yanguang \\ College of Information Systems \& Management \\ National University of Defense Technology \\ Changsha, Hunan, China
}

\author{
Zhu Yifan \\ College of Information Systems \& Management \\ National University of Defense Technology \\ Changsha, Hunan, China
}

\begin{abstract}
This paper mainly gives an efficient consistency algorithm for solving tigher solution space of Temporal Constraint Optimization Problem (TCOP), and it is a variation of Path Constraint $(\mathrm{PC})$ that is widely used in the domain of Constraint Satisfaction Problem (CPS). The algorithm can find out the feasible solutions and eliminate some non-feasible solutions, and it can improve the efficiency of solving TCOP. The validity and convergence of the algorithm are both proved by mathematical reasoning.
\end{abstract}

Keywords- Algorithm; Solution Space; Temporal Constraint Optimization Problem

\section{INTRODUCTION}

Temporal Constraint Optimization Problem(TCOP) is quite widely used in the problems such as time cooperation based combat projects optimization. So how to improve the effectiveness of TCOP optimizing computing is a significant research task. How to improve the efficiency of TCOP Optimization computing is a problem needed to solve. TCOP problem has two aspects: one is to make sure if TCOP has any feasible solutions, the other is to search the optimization solution of TCOP.This paper is aim to get a feasible solution from knowing any value of a variable, through compressing the solution space of TCOP. Thus this method can efficiently solve the first aspect problem, at the same time, it can quickly get feasible solutions of TCOP, improving the searching efficiency of optimization computing in the second aspect problem.

Temporal Constraint information in TCOP can be equally expressed as Temporal Constraint Satisfaction Problem(TCSP). TCSP is a common theoretic framework dealing with temporal constraint raised by Dechter and others in 1991 [5], and TCOP can be seen as problem expansion of TCSP after introducing aim function. Thereinto the TCSP that only include single temporal constraint is called Simple Temporal Problems (STP), and STP is a kind of TCSP widely studied and applied [6-7]. This paper mostly studies solving TCOP that can be expressed as STP. A classic STP can be described as the duality group below:

$$
\mathrm{STP}=\langle\mathrm{X}, \mathrm{C}\rangle
$$

$X=\{x 1, x 2, \ldots, x n\}$ means the set of time variable, $C$ means

the set of all the constraint conditions. Constraint conditions contain unitary constraint and duality constraint. Unitary constraint is defined as $\mathrm{li} \leq \mathrm{xi} \leq \mathrm{ui}$, expressing the value range of time variable; duality constraint is defined as lij $\leq x j-x i \leq$ uij, expressing the value range of time delay. Suppose $\mathrm{x} 0 \equiv 0, \mathrm{x} 0$ is time origin. When introducing time origin, unitary constraint can be equally defined as duality constraint $l i \leq x j-x 0 \leq$ ui. Thus all the constraint conditions in STP can be expressed as duality constraint.

If there is a group $\mathrm{T}=(\tau 1, \tau 2, \ldots, \tau \mathrm{n})$, when the values of time variables are $x 1=\tau 1,, x 2=\tau 2, \ldots, x n=\tau n$, it can satisfy all the constraint conditions in STP, then group $\mathrm{T}$ is called a set of feasible solutions of STP.

So TCOP in this paper can be expressed as TCOP $=\langle\mathrm{X}$, $\mathrm{C}, \mathrm{f}\rangle$, thereinto $\mathrm{f}$ means the optimized target of TCOP.

Sample Temporal Network (STN) is a efficient method to

describe and infer systems with temporal knowledge and temporal constraint, STN puts the idea of solving problems used in Chart Theroy into solving Temporal Constraint Satisfaction Problems, abroad used in checking and clearing up time conflict[8]. The peak of STN means time variable, and the border between the peaks means restriction relation between time variables. In this paper, firstly, we establish STN model of temporal constraint, then based on STN, Path Consistency(PC) in constraint satisfaction problem [9-10], presenting the method of solution space contraction of TCOP, and the validity and convergence of the method are both proved by mathematical reasoning, at last, through a test application, this method is validated that it can efficiently improve the efficiency of optimization computing.

\section{The Dual Temporal Constraint Network EXPRESS OF TEMPORAL CONSTRAINT IN TCOP}

Definition 1: Assume that $a$ and $b(a \leq b)$ are two real numbers, define set $A=\{a \leq t \leq b \wedge t \in R\}$, comletely order set $(A, \leq)$ is called period of time,experessed as $I=[$ $\mathrm{a}, \mathrm{b}$ ], in which a and $\mathrm{b}$ separately express $I$ 's starting time and ending time. When $\mathrm{a}=\mathrm{b}$, period of time degenerate as $\mathrm{a}$ time point,obviously $\mathrm{a} \leq \mathrm{t} \leq \mathrm{b} \Leftrightarrow \mathrm{t} \in \mathrm{I}=[\mathrm{a}, \mathrm{b}]$ 。

Suppose period of time I1 $=[\mathrm{a}, \mathrm{b}]$ and $\mathrm{I} 2=[-\mathrm{b},-\mathrm{a}]$, then we call I1 and I2 opposite period of time,marked as $I_{2}=I_{1}^{-1} \quad$ or $I_{1}=I_{2}^{-1}$

Definition 2:Present period of time Ix $=[\mathrm{ax}, \mathrm{bx}]$ and Iy $=[$ ay, by $]$, define period of time merger operation $\oplus$

$$
[\mathrm{ax}, \mathrm{bx}] \oplus[\mathrm{ay}, \mathrm{by}]=[\mathrm{ax}+\mathrm{ay}, \mathrm{bx}+\mathrm{by}]
$$

Especially for any period of time $\mathrm{I}, \mathrm{I} \oplus \varnothing=\varnothing, \varnothing \oplus \mathrm{I}=$ $\varnothing$.Obviously period of time merger operation satisfies 
combine law and exchange law.

Define continuum merger operation sign $\Sigma$

$$
\sum_{i=1}^{n} I_{i}=\sum_{1 \leq i \leq n} I_{i}=I_{1} \oplus I_{2} \cdots \oplus I_{n}
$$

Definition 3:Present period of time Ix $=[\mathrm{ax}, \mathrm{bx}]$ and Iy $=$ [ay, by], define period of time cross operation $\otimes$

$[a x, b x] \otimes[a y, b y]=[\max (a x, a y), \min (b x, b y)]$

When $\max (\mathrm{ax}, \mathrm{ay})>\min (\mathrm{bx}, \mathrm{by}),[\mathrm{ax}, \mathrm{bx}] \otimes[a y$, by $]$ $=\varnothing$.Especially for any period of time $\mathrm{I} \otimes \varnothing=\varnothing, \varnothing \otimes \mathrm{I}=$ $\varnothing$.Obviously period of time cross operation satisfies combine law and exchange law.

Define continuous cross operation sign $\Pi$

$$
\prod_{i=1}^{n} I_{i}=\prod_{1 \leq i \leq n} I_{i}=I_{1} \otimes I_{2} \cdots \otimes I_{n}
$$

Character 1: Present period of time $I$, for $I_{i}$, $i=1,2, \ldots, n$,if $\prod_{i=1}^{n} I_{i} \neq \varnothing$, then $\left(\prod_{i=1}^{n} I_{i}\right) \oplus I=\prod_{i=1}^{n}\left(I_{i} \oplus I\right)$. Prove: Let $I_{1}=\left[a_{1}, b_{1}\right], I_{2 \_n}=\prod_{i=2}^{n} I_{i}=\left[a_{2 \_n}, b_{2 \_n}\right]$

$$
\because \prod_{i=1}^{n} I_{i} \neq \varnothing \text { and cross operation satisfies combine law }
$$$$
\therefore I_{1} \otimes I_{2 \_n} \neq \varnothing
$$$$
\therefore \max \left(a_{1}, a_{2 \_n}\right) \leq \min \left(b_{1}, b_{2 \_n}\right)
$$$$
\therefore\left(I_{1} \otimes I_{2 \_n}\right) \oplus I=\left[\max \left(a_{1}, a_{2 \_n}\right)+a, \min \left(b_{1}, b_{2 \_n}\right)+b\right]
$$$$
\left(I_{1} \oplus I\right) \otimes\left(I_{2 \_n} \oplus I\right)=\left[\max \left(a_{1}+a, a_{2 \_n}+a\right), \min \left(b_{1}+b, b_{2 \_n}+b\right)\right]
$$$$
\because \max \left(a_{1}, a_{2 n}\right)+a=\max \left(a_{1}+a, a_{2 n}+a\right)
$$$$
\min \left(b_{1}, b_{2 n}\right)+b=\min \left(b_{1}+b, b_{2 n}+b\right)
$$$$
\therefore\left(I_{1} \otimes I_{2 \_n}\right) \oplus I=\left(I_{1} \oplus I\right) \otimes\left(I_{2 \_n} \oplus I\right) \Leftrightarrow
$$

$$
\left(\prod_{i=1}^{n} I_{i}\right) \oplus I=\left(I_{1} \oplus I\right) \otimes\left[\left(\prod_{i=2}^{n} I_{i}\right) \oplus I\right]
$$

Then in turns analyzing we can prove charater 1 comes into existence.

Definition 4: For a Temporal Constraint optimization problem TCOP $=\langle\mathrm{X}, \mathrm{C}, \mathrm{f}\rangle$, in which Temporal Constraint can be expressed as the weighted direction figure $\mathrm{DG}=\langle\mathrm{V}$, $\mathrm{E}, \mathrm{W}>$ below.

$V$ is node concourse, any node vi one by one maps temporal variable xi,especially $\mathrm{v} 0$ maps time origin $\mathrm{x} 0$; $\mathrm{E}$ and $\mathrm{W}$ express direction border and weight set, any constraint qualification of STP: lij $\leq x j-x i \leq u i j$, corresponding two direction borders eij from node vi to node $\mathrm{vj}$ and eji from node vj to node vi in set E, let period of time Iij $=[1 \mathrm{ij}$, uij], then the weight of eij and eji is respective Iij and $I_{j i}=I_{i j}^{-1}$.

DG is called Dual Temporal Constraint Network (DTCN), by all appearances for any feasible solution of TCOP $\mathrm{T}=(\tau 1, \tau 2, \ldots, \tau \mathrm{n})$, and any direction broder of DTCN eij, $\tau j-\tau i=I i j$.

Definition 5: Suppose that in DTCN $=\langle\mathrm{V}, \mathrm{E}, \mathrm{W}\rangle$ one of the gateways from node vs to node ve is pse $=v s v 1 \ldots v k v e$, one of the gateways from node ve to node vs is pes=vevk...vlvs, then we call pse and pes are opposite gateways about node vs and node ve. According to Definition 4, opposite gateways cannot but exist in pairs.

Definition 6: Suppose that in DTCN $=\langle\mathrm{V}, \mathrm{E}, \mathrm{W}\rangle$ one of the gateways from node vs to node ve is pse=vsv1...vkve,suppose the weight of direction border es 1 is Is1, the weight of eke is Ike, the weight of ei, $i+1$ is $\mathrm{Ii}, \mathrm{i}+1, \mathrm{i}=1,2, \ldots, \mathrm{k}-1$.Period of time $\mathrm{Ise}=\mathrm{Is} 1 \oplus \mathrm{I} 12 \oplus \ldots \oplus \mathrm{Ike}$ is called the distance of period of time of gateway pse. When node $\mathrm{vs}=\mathrm{ve}$, Ise is called the distance of period of time of loop pse.

Definition 7:Suppose that in DTCN $=\langle\mathrm{V}, \mathrm{E}, \mathrm{W}\rangle$ there are $\mathrm{m}$ basic gateways from node vs to node ve, suppose that the distance of period of time in the ith basic acess is Ise, $i$, period of time $I d, s e=I s e, 1 \otimes I s e, 2 \otimes \ldots \otimes I s e, m$ is called the shortest distance of period of time from node vs to node ve.

\section{III.METHOD OF COMPRSSING SOLUTION SPACE OF TCOP}

For Temporal Constraint optimization problem TCOP $=\langle\mathrm{X}, \mathrm{C}, \mathrm{f}\rangle$ compressing solution space, in fact it is to obtain a weighted direction figure $\mathrm{DG}=\langle\mathrm{V}, \mathrm{E}, \mathrm{W}\rangle$ which can satisfy the following two qualifications:

Firstly,for any feasible solution of TCOP $\mathrm{T}=(\tau 1$, $\tau 2, \ldots, \tau \mathrm{n})$, and any direction border ei $\mathrm{j}$ of $\mathrm{DG}$, satisfying $\tau j-\tau i \in I i j$, then period of time Iij is the weight of eij ;

Secondly, for any weight Iij of DG, if $\tau \in$ Iij, then there must exsit a group of feasible solutions of TCOP:T $=(\tau 1$, $\tau 2, \ldots, \tau n)$, satisfying $\tau j-\tau i=\tau$.

Weighted direction figure DG is called smallest temporal constraint network of TCOP, noted as minNet. Appraently minNet is a constrictive expressing model of feasible solution space in TCOP, deflate the value area of any weight in minNet, and it will necessarily lead the loss of feasible solutions of TCOP.

Theorem 1: In DTCN $=\langle\mathrm{V}, \mathrm{E}, \mathrm{W}\rangle$ which is according to TCOP $=\langle\mathrm{X}, \mathrm{C}, \mathrm{f}\rangle$, if there is a shortest distance of period of time from node vs to node ve that is $\mathrm{Id}, \mathrm{se}=\varnothing$, then TCOP don't have feasible solutions.

Prove: Suppose that TCOP has a feasible solution $\mathrm{T}=$ $(\tau 1, \tau 2, \ldots, \tau \mathrm{n})$. Suppose that there are $\mathrm{m}$ basic gateways from node vs to node ve in DTCN, the ith basic gateway is pse, $i=v s v i 1$ vi $2 \ldots$ vikve.

Suppose that $\mathrm{xs}=\tau \mathrm{s}, \mathrm{xe}=\tau \mathrm{e}, \mathrm{xij}=\tau \mathrm{ij}$ are feasible solutions segments according to nodes in pse,I; The weight according to es,il is Is,il, the weight according to eik,e is lik,e, and the weight according to eij, $\mathrm{ji}+1$ is $\mathrm{Iji}, \mathrm{ji}+1, \mathrm{j}=1,2, \ldots \mathrm{k}-1$.

Based on the definition of feasible solution, we can get: $\tau \mathrm{i} 1-\tau_{\mathrm{s}}=\mathrm{Is}, \mathrm{i} 1, \tau \mathrm{e}-\tau \mathrm{ik}=\mathrm{Iik}, \mathrm{e}, \tau \mathrm{ij}+1-\tau \mathrm{ij}=\mathrm{Iij}, \mathrm{ij}+1$. Suppose that the distance of period of time according to pes,I is Ise, $\mathrm{i}$

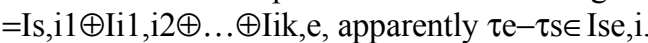

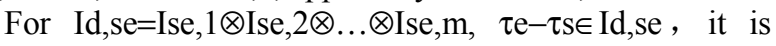
contrary to $\mathrm{Id}, \mathrm{se}=\varnothing$, then supposed qualification is not true, the original proposition is true.

Deduction 1: In DTCN $=\langle\mathrm{V}, \mathrm{E}, \mathrm{W}\rangle$ which is according to TCOP $=\langle\mathrm{X}, \mathrm{C}, \mathrm{f}\rangle$, if there is a basic loop whose distance of period of time I that don't contain 0 , i.e. $0 \notin \mathrm{I}$, then TCOP don't have feasible solutions.

Prove: Suppose any one basic gateway in DTCN is vs...vi...ve...vj...vs, accordingly, its distance of period of time is I, thereinto the distance of period of time of basic gateway vs...vi...ve is Ii $=[1 \mathrm{i}$, ui], the distance of period of time of basic gateway ve...vj...vs is $\mathrm{Ij}=[\mathrm{lj}$, uj], according to definition 6, I=Ii $\oplus \mathrm{Ij}=[\mathrm{li}+\mathrm{lj}$, ui $+\mathrm{uj}]$.

For $0 \notin \mathrm{I}, \quad \mathrm{i}+\mathrm{lj}>0$ or $\mathrm{ui}+\mathrm{uj}<0$.

When $1 \mathrm{i}+\mathrm{lj}>0$, according to definition 5 , the distance of 
period of time of the gateway opposite to ve...vj...vs is $I_{j}^{-1}=\left[-u_{j},-l_{j}\right]$

For $\quad u i \geq l i>-1 j, \quad \min (u i,-l j)=-1 j<l i ; \quad$ For $\quad u j \geq 1 j>-l i$, $\max (l i,-u j)=\quad$ li, and $\max (l i,-u j)>\min (u i,-l j)$, then $I_{i} \oplus I_{j}^{-1}=\varnothing$. According to definition 7 , the shortest distance of period of time from node vs to node ve is $\varnothing$, so according to theorem 1, TCOP don't have feasible solutions.

When ui+uj $<0$, in the same method we can prove that TCOP don't have feasible solutions.

Deduction 2: In DTCN $=\langle\mathrm{V}, \mathrm{E}, \mathrm{W}\rangle$ which is according to TCOP $=\langle\mathrm{X}, \mathrm{C}, \mathrm{f}\rangle$, any three different nodes $\mathrm{va}, \mathrm{vb}$ and $\mathrm{vc}$, thereinto the shortest distance of period of time from va to node $\mathrm{vc}$, from node $\mathrm{vc}$ to node $\mathrm{vb}$ and from node va to node $\mathrm{vb}$ are respectively $\mathrm{Id}, \mathrm{ac}, \mathrm{Id}, \mathrm{cb}$ and Id,ab , if TCOP has feasible solutions, then $\mathrm{Id}, \mathrm{ab} \subseteq \mathrm{Id}, \mathrm{ac} \oplus \mathrm{Id}, \mathrm{cb}$.

Prove: Suppose concourse Pac is made up of basic gateways from node va to node vc without node $v b$, and there are $m$ elements. Suppose that the distance of period of time of the ith basic gateway is Iac,I; Concourse Pcb is made up of basic gateways from node $\mathrm{vc}$ to node $\mathrm{vb}$ without node va, and there are $\mathrm{n}$ elements, the distance of period of time of the jth basic gateway is Icb,j; Concourse Pab is made up of basic gateways from node va to node vb without node $\mathrm{vc}$, and there are 1 elements, the distance of period of time of the kth basic gateway is Iab,k.

The definition of Descartes Product of concourse Pac and Pcb:

$P a c \times c b=\{v a \ldots v i \ldots v c \ldots v j \ldots v b \mid v a \ldots v i \ldots v c \in P a c \wedge v c \ldots v j$ $\ldots v b \in \mathrm{Pcb}\}$

Cross operation result of distance of period of time of All the gateways in Concourse Pac $\times c b$ is:

$$
I_{a c \times c b}=\prod_{i=1}^{m} \prod_{j=1}^{n}\left(I_{a c, i} \oplus I_{c b, j}\right)
$$

Suppose concourse Pacb is made up of basic gateways from node $\mathrm{va}$ to $\mathrm{vb}$ containing node $\mathrm{vc}$, for any va...vi...vc...vj...vbePacb, we can get one and only breaking up to two parts: va...vi...vc and $v c . . v \mathrm{vj} . . \mathrm{vb}$. Apparently va...vi...vcePac, $v c . . v j . . v b \in P c b$, so va...vi...vc...vj...vb $\in$ Pac $\times$ cb, and then Pacb $\subseteq$ Pac $\times$ cb. Suppose the continuous cross operation result of all the distance of period of time in concourse is Pacb, apparently Iac $\times$ cb $\subseteq$ Iacb.

The shortest distance of period of time from node va to node $\mathrm{vb}$ is : $I_{d, a b}=\prod_{k=1}^{l} I_{a b, k} \otimes I_{a c b}$.

Let period of time $I_{z, a b}=\prod_{k=1} I_{a b, k} \otimes I_{a c \times c b}$, they apparently satisfy inclusion relation: $\mathrm{I} z, \mathrm{ab} \subseteq \mathrm{Iab}$ 。

On the other hand, for any $\tau \in \mathrm{Id}$,ab, if $\tau \in \mathrm{Iz}, \mathrm{ab}$, then apparently iff $\tau \in \mathrm{Iij}, \mathrm{acb}$, Iij,acb is the distance of period of time of any nonbasic gateway $\mathrm{Pij}, \mathrm{acb}=\mathrm{va} . . . \mathrm{vi} . . . \mathrm{vc} \ldots \mathrm{vj} \ldots \mathrm{vb}$ in Pac $\times$ cb.

For va...vi...vc and $v c \ldots v j \ldots v b$ are both basic gateways, there are at least a pair of same nodes vt on both sides of node vc, so as to $\mathrm{Pij}, \mathrm{acb}=\mathrm{va} \ldots \mathrm{vi} \ldots \mathrm{vc} \ldots \mathrm{vj} \ldots \mathrm{vb}$, and $\mathrm{vt} . . \mathrm{vc} \ldots \mathrm{vt}$ is a basic loop. Let the distance of period of time of va...vt be Iat, the distance of period of time of $\mathrm{vt} . . . \mathrm{vc} . . \mathrm{vt}$ be Itt, the distance of period of time of vt...vb be Itb, then the distance of period of time of va...vt...vb is Iatb=Iat $\oplus \mathrm{Itb}$. Thus Iij, $\mathrm{acb}=\mathrm{Iat} \oplus \mathrm{Itt} \oplus \mathrm{Itb}=\mathrm{Iatb} \oplus \mathrm{Itt}$.

For TCOP has feasible solutions, based on deduction 1, $0 \in \mathrm{Itt}$, any $\tau \in \mathrm{Iatb}, \tau=\tau+0 \in \mathrm{Iatb} \oplus \mathrm{Itt}=\mathrm{Iij}, \mathrm{acb}$. Iatb $\subseteq \mathrm{Iij}$,acb.

If there is still some basic loops in va...vt...vb, then pick-up basic loops in turn, until basic gateway, then the basic gateway is apparently the basic gateway from node va to $\mathrm{vb}$ without node $\mathrm{vc}$, the worst is the basic gateway vavb. Suppose this basic gateway is $p k, a b, p k, a b \in P a b$, and the distance of period of time of $\mathrm{pk}, \mathrm{ab}$ is $\mathrm{Ik}, \mathrm{ab}$, then apparently $\mathrm{Ik}, \mathrm{ab} \subseteq \mathrm{Iij}, \mathrm{acb}$.

For $\tau \in \mathrm{Id}, \mathrm{ab}, \tau \in \mathrm{Ik}$,ab. For $\mathrm{Ik}, \mathrm{ab} \subseteq \mathrm{Iij}, \mathrm{acb}, \tau \in \mathrm{Iij}, \mathrm{acb}$, $\mathrm{Id}, \mathrm{ab} \subseteq \mathrm{Iz}, \mathrm{ab}$, and

$$
I_{d, a b}=I_{z, a b}=\prod_{k=1}^{l} I_{a b, k} \otimes\left(\prod_{i=1}^{m} \prod_{j=1}^{n}\left(I_{a c, i} \oplus I_{c b, j}\right)\right)
$$

Let period of time ${ }^{I_{a c}=\prod_{i=1}^{m} I_{i, a c}}$, period of time $I_{c b}=\prod_{j=1}^{n} I_{j, c b}$, period of time $I_{a b}=\prod_{k=1}^{l} I_{k, a b}$, for TCOP has feasible solutions, based on theorem 1 , Iac $\neq \varnothing, \operatorname{Icb} \neq \varnothing$, $\mathrm{Iab} \neq \varnothing$.

Based on definition 1, we can get:

$$
\begin{aligned}
& \prod_{i=1}^{m} \prod_{j=1}^{n}\left(I_{a c, i} \oplus I_{c b, j}\right) \\
& =\prod_{i=1}^{m} I_{a c, i} \oplus\left(\prod_{j=1}^{n} I_{c b, j}\right)=\prod_{i=1}^{m} I_{a c, i} \oplus I_{c b}=I_{a c} \oplus I_{c b}
\end{aligned}
$$

Thus $I_{d, a b}=I_{a b} \otimes\left(I_{a c} \oplus I_{c b}\right)$

In the same method we can prove that: $I_{d, a c}=I_{a c} \otimes\left(I_{a b} \oplus I_{c b}^{-1}\right)$ and $I_{d, c b}=I_{c b} \otimes\left(I_{a c}^{-1} \oplus I_{a b}\right)$.

For any $\tau \in I_{d, a b}$, apparently $\tau \in I_{a b}$ and $\tau \in I_{a c} \oplus I_{c b}$, so there is $\tau_{a} \in I_{a c}$ and $\tau_{c} \in I_{c b}$, satisfying $\tau_{a}+\tau_{c}=\tau$.

$$
\begin{aligned}
& \because-\tau_{c} \in I_{c b}^{-1} \text { and } \tau \in I_{a b}, \therefore \tau_{a}=\tau-\tau_{c} \in I_{a b} \otimes I_{c b}^{-1}, \\
& \therefore \tau_{a} \in I_{a c} \otimes\left(I_{a b} \oplus I_{c b}^{-1}\right), \text { i.e. } \tau_{a} \in I_{d, a c} . \\
& \because-\tau_{a} \in I_{a c}^{-1} \text { 且 } \tau \in I_{a b}, \quad \therefore \tau_{c}=-\tau_{a}+\tau \in I_{a c}^{-1} \oplus I_{a b}, \\
& \therefore \tau_{c} \in I_{c b} \otimes\left(I_{a c}^{-1} \oplus I_{a b}\right), \text { i.e. }{ }^{{ }_{c} \in I_{d, c b},} \\
& \therefore \tau=\tau_{a}+\tau_{c} \in I_{d, a c} \oplus I_{d, c b}, \quad \therefore I_{d, a b} \subseteq I_{d, a c} \oplus I_{d, c b} .
\end{aligned}
$$

Theorem 2: Suppose weighted direction figure $\mathrm{DG}=\langle$ $\mathrm{V}, \mathrm{E}, \mathrm{W}\rangle$ is a complete figure, Dual Temporal Constraint Network according to TCOP $=\langle\mathrm{X}, \mathrm{C}, \mathrm{f}\rangle$ is DTCN $=\langle\mathrm{V}, \mathrm{E}$, $\mathrm{W}\rangle$, thereinto the weight of direction border eij in DG is the shortest distance of period of time from node vi to $\mathrm{vj}$ in DTCN, then weighted direction figure DG is the minimum Temporal Constraint Network of TCOP.

Prove: Firstly, we prove that qualification 1 is true.

Suppose the feasible solution of TCOP is $\mathrm{T}=$ $(\tau 1, \tau 2, \ldots, \tau \mathrm{n})$, considering the weight of any direction border eij of DG: Id,ij, and there are $m$ basic gateways from node vi to $\mathrm{vj}$ in DTCN, the kth basic gateway is pk=vivk1vk2 ...vklvj.

Suppose the feasible solutions segments according to the 
nodes in $p k$ are : $x i=\tau i, x j=\tau j, x k t=\tau k t$; The weight according to ei,k1 is Ii,k1, the weight according to ekl, jis Ikl.j, the weight according to ekt,kt+1 is Ikt,kt+1, $\mathrm{t}=1,2, \ldots, 1-1$.

Based on the definition of feasible solutions:

$\tau \mathrm{k} 1-\tau \mathrm{i}=\mathrm{Ii}, \mathrm{k} 1, \tau \mathrm{j}-\tau \mathrm{kl}=\mathrm{Ikl}, \mathrm{j}, \tau \mathrm{kt}+1-\tau \mathrm{kt}=\mathrm{Ikt}, \mathrm{kt}+1$

The distance of period of time of $\mathrm{pk}$ is: $\mathrm{Iij}, \mathrm{k}=\mathrm{Ii}, \mathrm{k} 1 \oplus \mathrm{Ik} 1, \mathrm{k} 2 \oplus \ldots \oplus \mathrm{Ikl}, \mathrm{j}, \quad$ apparently $\quad \tau \mathrm{j}-\tau \mathrm{i}=\mathrm{Iij}, \mathrm{k} . \quad$ For $\mathrm{Id}, \mathrm{ij}=\mathrm{Iij}, 1 \otimes \mathrm{Iij}, 2 \otimes \ldots \otimes \mathrm{Iij}, \mathrm{m}, \quad$ apparently $\quad \tau \mathrm{j}-\tau \mathrm{i}=\mathrm{Id}, \mathrm{ij}$, qualification 1 is true.

Secondly, we prove that qualification 2 is true.

Considering the weight of direction border e12 in DG: Id, 12, suppose any $\tau \in \mathrm{Id}, 12$, we prove that there is a group of feasible solutions of TCOP: $\mathrm{T}=(\tau 1, \tau 2, \ldots, \tau \mathrm{n})$, satisfying $\tau 2-\tau 1=\tau$.

Based on deduction 2, $I_{d, 12} \subseteq I_{d, 10} \oplus I_{d, 02}=I_{d, 01}^{-1} \oplus I_{d, 02}$,

Thus there is $\tau 2-\tau 1=\tau$ so that $\tau 1 \in \mathrm{Id}, 01$ and $\tau 2 \in \mathrm{Id}, 02$. Apparently when $\mathrm{x} 1=\tau 1, \mathrm{x} 2=\tau 2$, satisfying all the restrictions among the time varibles $\mathrm{x} 0, \mathrm{x} 1, \mathrm{x} 2, \mathrm{x} 0 \equiv 0$.

We expand $\mathrm{x} 1=\tau 1, \mathrm{x} 2=\tau 2$ into a feasible solution through mathematic induction:

When $\mathrm{k}=2, \quad \mathrm{x} 1=\tau 1, \ldots, \mathrm{xk}=\tau \mathrm{k} \quad$ apparently satisfy $\tau j-\tau i \in I d, i j, \quad 0 \leq i \neq j \leq 2$.Suppose when $\quad k=m, \quad m \geq 2$, $\mathrm{x} 1=\tau 1, \ldots, \mathrm{xk}=\tau \mathrm{k}$ satisfy $\tau \mathrm{j}-\tau \mathrm{i} \in \mathrm{Id}, \mathrm{ij}, \quad 0 \leq \mathrm{i} \neq \mathrm{j} \leq \mathrm{m}$.

When $\mathrm{k}=\mathrm{m}+1$, suppose there is a node $\tau_{\mathrm{V}}$ satisfying $\tau_{\mathrm{v}}-\tau \mathrm{i} \in \mathrm{Id}, \mathrm{ik}, \quad 0 \leq \mathrm{i} \leq \mathrm{m}$. Let Id,ik=[lik, uik], then $\tau \mathrm{i}+$ lik $\leq \tau_{\mathrm{V}}$ $\leq \tau i+$ uik.

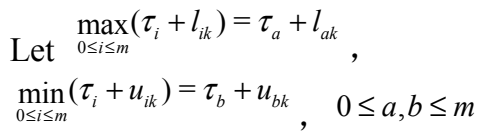

If $a=b$, then apparently $\max _{0 \leq i \leq m}\left(\tau_{i}+l_{i k}\right) \leq \min _{0 \leq i \leq m}\left(\tau_{i}+u_{i k}\right)$.

If $a \neq b$, then based on qualification we can suppose that $\tau_{b}-\tau_{a} \in I_{d, a b}$

Based on deduction 2, we can get: $I_{d, a b} \subseteq I_{d, a k} \oplus I_{d, k b}=I_{d, a k} \oplus I_{d, b k}^{-1}$

$\therefore \tau_{b}-\tau_{a} \in I_{d, a k} \oplus I_{d, b k}^{-1}, \therefore \tau_{b}-\tau_{a} \geq l_{a k}-u_{b k}$

$\therefore \tau_{a}+l_{a k} \leq \tau_{b}+u_{b k}, \quad \therefore \max _{0 \leq i \leq m}\left(\tau_{i}+l_{i k}\right) \leq \min _{0 \leq i \leq m}\left(\tau_{i}+u_{i k}\right)$

Thus the time node $\tau_{\mathrm{V}}$ that satisfies $\tau_{\mathrm{V}}-\tau_{\mathrm{i}} \in \mathrm{Id}$, ik $(0 \leq \mathrm{i} \leq \mathrm{m})$ exsits, let $\tau \mathrm{m}+1=\tau_{\mathrm{V}}$, then there are $\mathrm{x} 1=\tau 1, \ldots, \mathrm{xk}=\tau \mathrm{k}$ that satisfy $\tau \mathrm{j}-\tau \mathrm{i} \in \mathrm{Id}, \mathrm{ij}, 0 \leq \mathrm{i} \neq \mathrm{j} \leq \mathrm{m}+1$. So when $\mathrm{k}=\mathrm{n}, \mathrm{T}=(\tau 1, \tau 2, \ldots, \tau \mathrm{n})$ is a feasible solution of TCOP, and qualification 2 is true.

Based on theorem 2 , we know that as long as we can figure out the shortest distance of period of time among all the nodes in Dual Temporal Constraint Network: DTCN $=\langle\mathrm{V}, \mathrm{E}, \mathrm{W}\rangle$, then we can obtain the minimum Temporal Constraint Network of TCOP. The table I below shows when we already know the value of some varibles, the Feasible Solutions Extend Arithmetic of TCOP.

If feasible extend arithmetic has validity and astringency, then we must assure the value of $x k$ in the first step is not $\varnothing$. Based on the provement of theorem 2, inequation $\max _{0 \leq i<k}\left(\tau_{i}+l_{i k}\right) \leq \min _{0 \leq i<k}\left(\tau_{i}+u_{i k}\right)$ must be true, so feasible extend arithmetic is apparently efficient, and it can efficiently produce the feasible solutions of TCOP.
TABLE I. FeAsibLE SOLUTIONS EXTEND ARITHMETIC DESCRIPTION

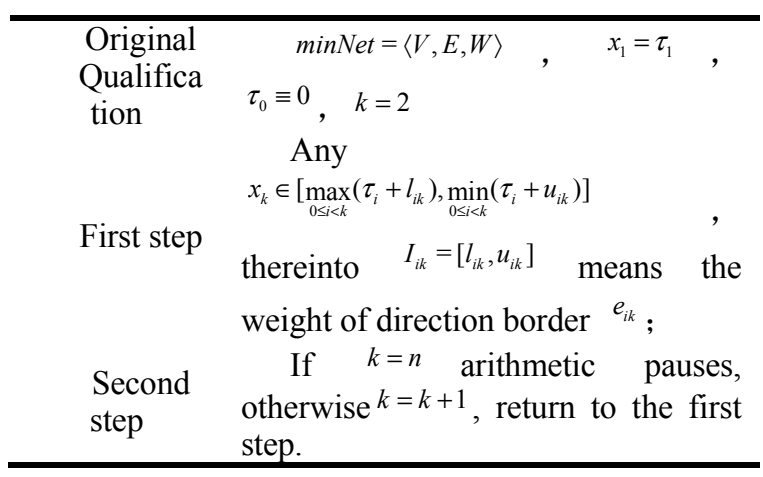

\section{IV.APPLICATIONS}

Consider a TCOP containing 5 time varibles as below:

$$
\left\{\begin{array}{l}
\max f\left(t_{1}, t_{2}, t_{3}, t_{4}, t_{5}\right)=t_{1}^{2}+t_{2}^{2}+t_{3}^{2}+t_{4}^{2}+t_{5}^{2} \\
\text { s.t. }\left\{\begin{array}{l}
0 \leq t_{2}-t_{1} \leq 5,5 \leq t_{4}-t_{3} \leq 10,5 \leq t_{3}-t_{1} \leq 10, \\
0 \leq t_{5}-t_{2} \leq 5,0 \leq t_{5}-t_{3} \leq 5,5 \leq t_{4}-t_{1} \leq 10 \\
0 \leq t_{1} \leq 5, t_{2}, t_{3}, t_{4}, t_{5} \geq 0
\end{array}\right.
\end{array}\right.
$$

Using restriction measure-changed method, the optimized solution through 12 times iteration is: $\mathrm{t} 1=5, \mathrm{t} 2=10$, $\mathrm{t} 3=10, \mathrm{t} 4=15, \mathrm{t} 5=15$.

Dual Temporal Constraint Network of TCOP is shown as below in Figure 1:

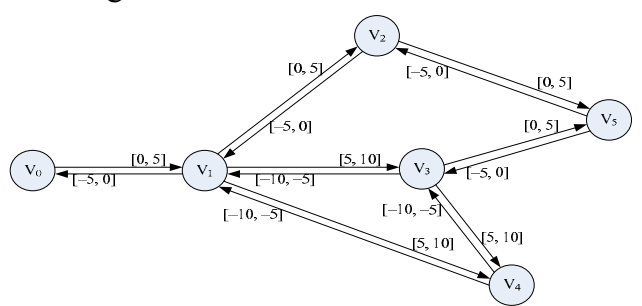

Figure 1. DTCN

As Figure 1 shows, node and time variable are cooresponding one by one, thereinto node $\mathrm{V} 0$ is cooresponding to the time origin.

There are three basic gateways from node V1 to V5: $\mathrm{P} 1=$ $\mathrm{V} 1 \mathrm{~V} 2 \mathrm{~V} 5, \mathrm{P} 2=\mathrm{V} 1 \mathrm{~V} 3 \mathrm{~V} 5, \mathrm{P} 3=\mathrm{V} 1 \mathrm{~V} 4 \mathrm{~V} 3 \mathrm{~V} 5$. The distance of period of time of three basic gateways are $\mathrm{I} 15,1=[0,5] \oplus[0,5]=[0,10], \quad \mathrm{I} 15,2=[5,10] \oplus[0,5]=[5,15] \quad$ and $\mathrm{I} 15,3=[5,10] \oplus[-10,-5] \oplus[0,5]=[-5,10]$, so the least distance of period of time from node $\mathrm{V} 1$ to $\mathrm{V} 5$ is: $\mathrm{Id}, 15=[0,10] \otimes[5,15] \otimes[-5,10]=[5,10]$.

Through computing the smallest distance of period of time among all the nodes in the Dual Temporal Constraint Network as shown in Figure 1, we can get the smallest temporal constraint network of TCOP, its weight value is shown as Table II.

TABLE II. WEIGHT OF MINIMUM TEMPORAL CONSTRAINT NETWORK

\begin{tabular}{|l|l|l|l|l|l|l|}
\hline & V0 & V1 & V2 & V3 & V4 & V5 \\
\hline V0 & {$[0,0]$} & {$[0,5]$} & {$[0,10]$} & {$[5,10]$} & {$[10,15]$} & {$[5,15]$} \\
\hline V1 & {$[-5,0]$} & {$[0,0]$} & {$[0,5]$} & {$[5,5]$} & {$[10,10]$} & {$[5,10]$} \\
\hline V2 & {$[-10,0]$} & {$[-5,0]$} & {$[0,0]$} & {$[0,5]$} & {$[5,10]$} & {$[0,5]$} \\
\hline V3 & {$[-10,-5]$} & {$[-5,-5]$} & {$[-5,0]$} & {$[0,0]$} & {$[5,5]$} & {$[0,5]$} \\
\hline
\end{tabular}




\begin{tabular}{|l|l|l|l|l|l|l|}
\hline V4 & {$[-15,-10]$} & {$[-10,-10]$} & {$[-10,-5]$} & {$[-5,-5]$} & {$[0,0]$} & {$[-5,0]$} \\
\hline V5 & {$[-15,-5]$} & {$[-10,-5]$} & {$[-5,0]$} & {$[-5,0]$} & {$[0,5]$} & {$[0,0]$} \\
\hline
\end{tabular}

From Table 2 we can find out that $\mathrm{t} 3=\mathrm{t} 1+5, \mathrm{t} 4=\mathrm{t} 1+$ 10, so TCOP above can predigest as below:

$$
\left\{\begin{array}{l}
\max f\left(t_{1}, t_{2}, t_{5}\right)=3 t_{1}^{2}+t_{2}^{2}+t_{5}^{2}+30 t_{1}+125 \\
\text { s.t. }\left\{\begin{array}{l}
0 \leq t_{2}-t_{1} \leq 5,0 \leq t_{5}-t_{2} \leq 5,5 \leq t_{5}-t_{1} \leq 10 \\
0 \leq t_{1} \leq 5,0 \leq t_{2} \leq 10,5 \leq t_{5} \leq 15
\end{array}\right.
\end{array}\right.
$$

When $\mathrm{t} 1=0$, based on Feasible Solutions Extend Arithmetic, we can extend a original feasible solution:

$$
\mathrm{t} 1=0, \mathrm{t} 2=5, \mathrm{t} 3=5, \mathrm{t} 4=10, \mathrm{t} 5=10
$$

Then using restriction measure-changed method, the optimized solution through 5 times iteration is:

$$
\mathrm{t} 1=5, \mathrm{t} 2=10, \mathrm{t} 3=10, \mathrm{t} 4=15, \mathrm{t} 5=15 \text {. }
$$

Apparently through the solution space contraction method of TCOP, we can efficiently predigest the searching range of feasible solution space when we optimizedly solve TCOP, therefore improving the efficiency of TCOP solving.

If we add two temporal constraints in the TCOP above: $5 \leq \mathrm{t} 3-\mathrm{t} 2 \leq 10,0 \leq \mathrm{t} 5-\mathrm{t} 4 \leq 5$, then the according Dual Temporal Constraint Network is shown as Figure 2:

From Figure 2 we can find that the period of time distance of basic loop $\mathrm{P}=\mathrm{V} 2 \mathrm{~V} 3 \mathrm{~V} 4 \mathrm{~V} 5 \mathrm{~V} 2$ is $\mathrm{I}=[5,10] \oplus[5,10] \oplus[0,5] \oplus[-5,0]=[5,20]$. Obviously $0 \notin \mathrm{I}$, thus based deduction 1, TCOP added restriction has no feasible solutions.

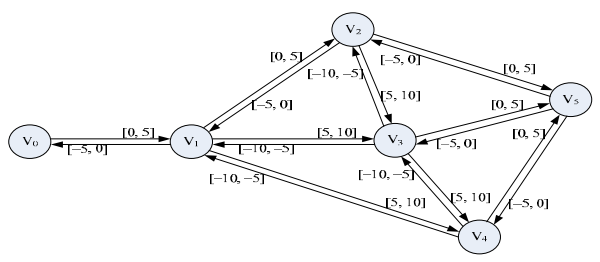

Figure2. DTCN added restriction

\section{CONCLUSIONS}

This paper proposes a method of compressing space of TCOP based on the method of Path Restriction Consistency resolving in restriction optimization problems resolving. The validity and convergence of the method are both strictly proved by mathematical reasoning. And the applications prove that this method can efficiently improve the efficiency of optimized computing.

\section{ACKNOWLEDGMENT}

The authors would like to gratefully acknowledge the financial support of the National Natural Science Foundation of China (No. 91024015 and No. 61074107).

\section{REFERENCES}

[1] S.X.Liu,Z.Guo,J.F.Tang. Constraint spread arithmetic of cumulation attemper problems with priority relation. Robotization Transaction, 2010,36(4):603-609(in Chinese).

[2] X.Huang,T.F.Yu. Project implement conflict recognise Arithmetic based Temporal Constraint Network. Chinese Management Science, 2008,16:197-201.

[3] Andreas F. Wehowsky. Safe distributed coordination of heterogeneous robots through dynamic simple temporal networks. Master's thesis, MIT, Cambridge, MA, 2003.

[4] Peter J. Schwartz and Martha E. Pollack. Planning with disjunctive temporal constraints. In Proceedings of the Nineteenth National Conference on Artificial Intelligence (AAAI-2004), 2004.

[5] Dechter R, Meiri I, Pearl J. Temporal constraint networks. Artificial Intelligence, 1991, 49(1-3):61-95

[6] Lin XU and Berthe Y. CHOUEIRY, A New Efficient Algorithm for Solving the Simple Temporal Problem. In Proceedings of the 10th International Symposium on Temporal Representation and Reasoning and Fourth International Conference on Temporal Logic, 2003.

[7] Amedeo Cesta, Angelo Oddi, and Stephen Smith. A constraint based method for project scheduling with time windows. Journal of Heuristics, 2002, 8(1):109-136

[8] L.H.Tang, C.Zhu, J.F.Luo. STN-based Time Conflict and Clear up. Computer Engineering, 2011, 37(3):251-253(in Chinese).

[9] A.K. Mackworth, Consistency in networks of relations, Artificial Intelligence. 1977, 8(1):99-118.

[10] A.K. Mackworth and E.C. Freuder, The complexity of some polynomial network consistency algorithms for constraint satisfaction problems, Artificial Intelligence. 1985, 25(1):65-74 